\title{
Commentary
}

\section{JHEAL: What Does It Mean to Initiate a New Journal?}

\author{
James F. Sallis, Ph.D. \\ University of California San Diego, USA \\ Australian Catholic University, Melbourne, Australia
}

To begin, I congratulate Jay Maddock, Ph.D., on the establishment of a new journal and a new publishing company. Though editing a journal is a big job, it is familiar territory for researchers. Starting a publishing company is a business enterprise almost all academics avoid. This will be a fascinating experiment to follow, because JHEAL (Journal of Healthy Eating and Active Living) signals a next step in the evolution of the turbulent field of academic publishing. The turbulence I refer to includes the rapid decline of hard copy journals, dramatically reduced publication lags, rise of authors paying publication fees, proliferation of low-quality "predatory" journals, decreasing reliance on libraries for journal access, large profits of some academic publishing companies, increasing ability to provide supplemental materials, and seemingly universal difficulty of recruiting qualified reviewers. Some of these trends are positive, some are negative, and others are just confusing. I don't pretend to know where academic publishing is heading or what an optimal model would be, but I'm confident that evolution will continue. Is this researcher-owned publishing company the start of a new trend?

Though JHEAL is part of the larger evolution of academic publishing, it is built on its own origin story. As described to me by Dr. Maddock, JHEAL came out of the paradigm shift promoted by Active Living Research (ALR; 2001-2016; Sallis et al., 2014) and Healthy Eating Research (HER; 2004-present; Lott et al., 2019; Story et al., 2006), both grant-funding programs supported by the Robert Wood Johnson Foundation. JHEAL not only pays tribute to these programs by adopting their names, JHEAL is designed to build on their legacies. ALR and HER shared primary goals. The first goal was to build evidence about policy and environmental drivers and interventions for their respective behavioral outcomes. This approach was intended to complement, and even remediate the limitations of, the dominant research focus on psychosocial influences on behavior and interventions targeting individuals and small groups. Broadening the focus of research and practice was expected to lead to multilevel interventions with enhanced reach and permanence of outcomes. A second goal of both programs was to support interdisciplinary research teams, because the greater complexity of the studies required a wider range of concepts, methods, and skills. Part of this goal was to support investigators with diverse personal backgrounds, to enhance the relevance of studies to vulnerable populations. A third common goal was to fund studies with the potential to influence policy and practice, then facilitate investigators to take actions to achieve that goal. Both programs appeared to have a rapid influence on behavioral research, based on an analysis of trends in policy and environment content of Society of Behavioral Medicine abstracts (Sallis et al., 2013). An independent evaluation of ALR documented progress on all the goals (Barker \& Gutman, 2014).

The goals and methods of JHEAL are consistent with those of ALR and HER. Submissions are preferred that deal with environmental, policy-relevant, and multilevel studies and interventions. Interdisciplinary studies are welcomed and prioritized. This is a significant distinction, because during a panel discussion of journal editors from various disciplines at an ALR conference, all editors stated their decisions were driven by serving their core disciplinary readers. None mentioned an emphasis on interdisciplinary papers. JHEAL is committed to contributing to the translation of research to practice and policy. The Journal is taking a risk by having sections for research and for practice or policy papers, but this practice will help expose these distinct audiences to material they would otherwise be unlikely to encounter.

These goals and methods are not unique to JHEAL, but they are unusual enough that I expect the focus of JHEAL will be a strength in attracting high-quality papers with appeal across multiple disciplines and solid potential for application. Policy, environmental, and multilevel research; interdisciplinary collaborations; international research; and translating research to practice and policy all remain challenges for researchers, and I believe JHEAL's focus will contribute to growing, strengthening, and sustaining this highly diverse community of researchers, practitioners, policy makers, and advocates.

$J H E A L$ is adopting practices likely to enhance its value and impact. For a fee-based journal, there are several features that improve its accessibility compared to other journals. The fees are lower than any journal I am aware of that requires fees, and there are several discount programs, including for submissions from low-income countries. Digital media files are encouraged, because maps, animations, infographics, audio files, and other materials should improve communication of findings and recommendations to diverse audiences. Commentaries, editorials, and audio summaries will deepen discussions and expand audiences. This first issue contains a special section, and I encourage similar themed sections or issues to become regular features because they become valuable collections of information on the current status of a research, practice, policy, or translation issue.

A bottom line question is, "Is a new journal really needed?" The answer is probably "no" because active living research and healthy eating research are already 
flourishing. But if the question is "Can JHEAL make important contributions?" my answer is "yes." The focus on applied research, the diversity of paper types, the inclusion of practice and policy papers, the interdisciplinary orientation, and the joint emphasis on active living and healthy eating - which are major drivers of numerous leading causes of death - are all agendas that can and should be nurtured. Inactive lifestyles, unhealthy diets, and the related chronic diseases remain among the biggest challenges in global public health. The launch of JHEAL during a historic pandemic is responsive to current needs, because the COVID-19 pandemic has brought new awareness of the important contributions of these challenges to infectious disease outcomes. Horton (2020) explains how COVID-19, chronic diseases, and sociodemographic inequalities constitute a syndemic because they are all interrelated. The current situation brings new urgency to advance and expand active living and healthy eating research while improving its translation to even broader domains of practice and policy.

Corresponding author:

James F. Sallis, Ph.D.

Distinguished Professor Emeritus

Herbert Wertheim School of Public Health and Human

Longevity Science

University of California, San Diego, MC 0631

9500 Gilman Drive. La Jolla, CA 92093-0631 USA

Email: jsallis@ucsd.edu

https://orcid.org/0000-0003-2555-9452

\section{Creative Commons License:}

This work is licensed under a Creative Commons Attribution-Noncommercial 4.0 International License (CC BY-NC 4.0).
Because an experienced investigator is leading both the Journal and the publishing company, I expect JHEAL itself will evolve, be open to thoughtful input, and be willing to innovate. I begin that process here by encouraging the editors to solicit, and investigators to submit, papers on the roles of active living and healthy eating in the syndemic involving COVID-19, chronic diseases, and sociodemographic inequality. In addition, it is increasingly vital for our healthy eating and active living research to make explicit connections with the threat of climate change. There is tremendous opportunity for improvements in movement and eating patterns to contribute to reducing greenhouse gases while enhancing health. As we understand better how our topics of active living and healthy eating are promising remedies not only for chronic diseases, but also social injustices, infectious diseases, and even the health of the planet, the need for actionable evidence becomes ever more pressing. With this new journal, let's be bold in what we try to accomplish.

Suggested citation (APA 7th edition): Sallis, J. F. (2020). JHEAL: What does it mean to initiate a new journal? Journal of Healthy Eating and Active Living, 1(1), 1-2.

\section{References}

Barker, D. C., \& Gutman, M. A. (2014). Evaluation of Active Living Research: Ten years of progress in building a new field. American Journal of Preventive Medicine, 46(2), 208-215.

Horton, R. (2020). COVID-19 is not a pandemic. The Lancet, 396 (September), 874.

Lott, M., \& Story, M. (2019). The future of Healthy Eating Research. In Healthy Eating, Active Living Supplement to Stanford Social Science Review. Summer 2019. http://stanford.ebookhost.net/ssir/digital/63/ebook/1/download.pdf

Sallis, J. F., Carlson, J. A., Mignano, A. M., Lemes, A., \& Wagner, N. (2013). Trends in presentations of environmental and policy studies related to physical activity, nutrition, and obesity at Society of Behavioral Medicine, 1995-2010: A commentary to accompany the Active Living Research Supplement to Annals of Behavioral Medicine. Annals of Behavioral Medicine, 45(1, suppl 1), 14-17.

Sallis, J. F., Cutter, C. L., Lou, D., Spoon, C., Wilson, A. L., Ding, D., Ponkshe, P., Cervero, R. Patrick, K., Schmid, T. L., Mignano, A., \& Orleans, C. T. (2014). Active Living Research: Creating and using evidence to support childhood obesity prevention. American Journal of Preventive Medicine, 46(2), 195-207.

Story, M., \& Orleans, C. T. (2006). Building evidence for environmental and policy solutions to prevent childhood obesity: The healthy eating research program. American Journal of Preventive Medicine, 30(1), 96-97. 